\title{
Percepción de la Calidad Educativa y su Relación con la Postulación a la Universidad Nacional Jorge Basadre Grohmann Tacna, 2008
}

\begin{abstract}
RESUMEN En tiempos donde la modernidad y la competitividad se hacen presentes, también constituye un desafio para las instituciones de Educación Superior el desplegar estrategias internas y externas para mantener su vigencia, y poder cumplir con el rol asignado a la Universidad, que es formar profesionales que la sociedad necesita.
\end{abstract}

La región Tacna, cuenta además de la Universidad Nacional Jorge Basadre Grohmann(UNJBG) con otras instituciones de Educación Superior, lo que indica que la sociedad estudiantil tiene otras opciones para acceder a la educación superior, por lo que el presente trabajo de investigación estuvo orientado a indagar la percepción de la calidad educativa y cómo esta se relaciona con la postulación a nuestra casa superior de estudios.

Los resultados de la percepción de la calidad educativa de la sociedad tacneña es favorable, ya que la UNJBG mantiene su vigencia por la imagen y prestigio ganado a través de su trayectoria como la primera casa de estudios en la región, considera que tiene personal docente capacitado, experiencia en la formación profesional. Cuenta con la infraestructura y equipamiento adecuados, que garantizan la educación. En relación con la postulación, la UNJBG brinda mayor oportunidad de postulación por la diversidad de carreras profesionales.

Llama la atención que para la sociedad no son indiferentes las ocurrencias de la problemática propia de una Universidad estatal, a lo cual está atenta, y que como producto de esta investigación queda como desafio para la UNJBG formular las politicas internas y externas que permitan mejorar dicho aspecto, pues si no se tiene un buen manejo, podria ser una amenaza para su vigencia y preferencia de postulación.

\begin{abstract}
In times where the modernity and the competitiveness become present, also they constitute a challenge for the Institutions of Top Education to open(deploy) internal and external strategies to support his(her,your) force, and to be able to expire with the role assigned to the University, that it(he,she) is to form(train) professionals that the society needs.
\end{abstract}

The region Tacna, account(bill) besides the National University Jorge Basadre Grohmann with other institutions of Top Education, which indicates that the student society has other options to accede to the top education, by what the present work of investigation(research) was orientated to investigate the perception of the educational quality and as this one it(he,she) relates to the postulation to our top house of studies.

The results of the perception of the educational quality of the society tacneña, it (he,she) is favorable, since the UNJBG supports his(her,your) force for the image and prestige gained(earned) across his(her,your) path as the first university in the region, thinks that it(he,she) has educational qualified personnel, experience in the vocational training and that relies on the infrastructure and suitable equipment(equipping), that it(he,she) guarantees the education. In relation to the postulation, the UN.JBG offers major opportunity of postulation for the diversity of professional careers(races).

It(He,She) calls the attention that for the society is not indifferent the occurrences of the own(proper) problematics of a state University, to which it(she) is attentive, and that as product of this investigation(research) stays as challenge for the UNJBG; to formulate the internal and external policies that allow to improve the above mentioned aspect, since if a good managing is not had, it might be a threat for his(her,your) force and preference of postulation.
INTRODUCCIÓN La Universidad Nacional Jorge Basadre Grohmann se rige por la base legal de la Educación Superior en nuestro país, la Ley Universitaria $\mathrm{N}^{\mathrm{o}} 23733$, y el Estatuto y Reglamento de la UNJBG, donde se considera que la principal función de la Universidad es la difusión del saber, la ciencia y la cultura, así como el desarrollo de la investigación y de la proyección y extensión universitario. La Ley y el Estatuto son claros al indicar que es el Docente el encargado de la Educación y, por ende, la imagen de la Universidad está dada por la calidad de Docentes y ello conlleva a la calidad educativa que se brinda en nuestra
Casa Superior de Estudios.

La Universidad como institución tiene la ardua tarea de reforzar y apoyar la cantidad y calidad de los medios efectivos para esta formación de manera más fluida y con variedad de opciones.

Nuestra Universidad cuenta con 15 Facultades y 29 escuelas profesionales; siendo la que promueve mayores opciones y variedad de carreras en la región.

A la pregunta ¿cuál es la percepción de la calidad educativa de la UNJBG y en qué medida esta percepción 
influye en la decisión de postulación a esta institución?, encontramos que tenemos muchas fortalezas para continuar brindando educación de calidad, pero al mismo tiempo debilidades que debemos tomar en cuenta para mantener nuestra vigencia social y de acreditación como tal.

\section{OBJETIVOS}

1. Describir la percepción que la sociedad tiene sobre la calidad educativa de la Universidad Nacional Jorge Basadre Grohmann.

2. Identificar aspectos que motivan la postulación de estudiantes a la Universidad Nacional Jorge Basadre Grohmann.

3. Establecer la relación entre la percepción de la calidad educativa y la postulación a la Universidad Nacional Jorge Basadre Grohmann.

\section{HIPÓTESIS}

La percepción de la calidad educativa de la Universidad Nacional Jorge Basadre Grohmann es buena, lo que la hace elegible para postulación de estudiantes.

\section{VARIABLES}

\section{Variable Independiente}

Percepción de la calidad educativa.

\section{Variable Dependiente}

Postulación a la Universidad Nacional Jorge Basadre Grohmann.

\section{Diseño de la Investigación}

La presente investigación es de tipo descriptivo y transversal; descriptivo porque nos permitirá determinar cómo está o cómo es el comportamiento o la situación de las variables de estudio en la población seleccionada; $y$ transversal porque se tomará en un periodo único y breve de tiempo delimitado para determinar la interacción de los factores psicosociales.

\section{MÉTODOSYTÉCNICAS}

Para la recolección de datos se utilizó el método de muestreo estratificado con afijación proporcional por Facultades, para el caso de la selección de la unidad de análisis se utilizó el muestreo simple aleatorio. Se utilizó un instrumento estructurado con 20 ítems, que además consideró datos generales y específicos.
Cuadro $\mathrm{N}^{\circ} 4$ Distribución según percepción que tiene la sociedad con respecto a la UNJBG Tacna 2008

\begin{tabular}{|c|c|c|c|}
\hline Percepción de la sociedad & $\operatorname{Res}$ & estas & $\begin{array}{l}\text { Porcentaje de } \\
\text { casos }\end{array}$ \\
\hline Criterios & $N^{N}$ & Porcentaje & $\mathrm{N}^{*}$ \\
\hline Desacuerdo & 173 & $7,5 \%$ & $45,1 \%$ \\
\hline Parcialmente de acuerdo 2 & 406 & $17,6 \%$ & $105,7 \%$ \\
\hline De acuerdo & 1017 & $44,1 \%$ & $264,8 \%$ \\
\hline Muy de acuerdo & 553 & $24,0 \%$ & $144,0 \%$ \\
\hline Totalmente de acuerdo 5 & 155 & $6,7 \%$ & $40,4 \%$ \\
\hline & & & \\
\hline
\end{tabular}

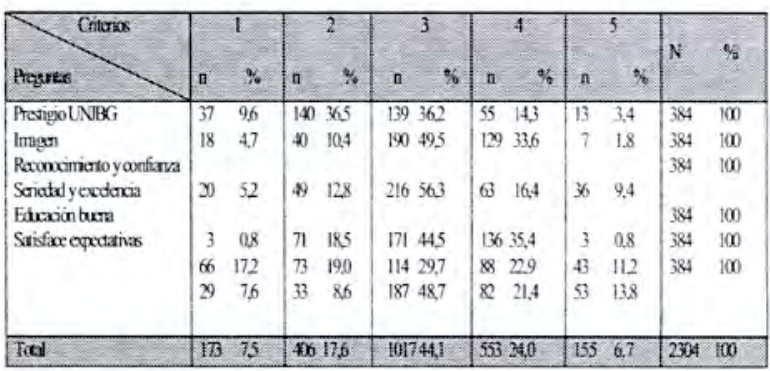

Fuente: Encuesta Dic. 2008-Febrero 2009

\section{Interpretación}

Observamos que el $44,1 \%$ de los estudiantes está de acuerdo y percibe a la Universidad Nacional Jorge Basadre Grohmann como una Institución de prestigio, con imagen, de reconocimiento y confianza por parte de la sociedad, con seriedad y excelencia, que brinda una Educación de buena calidad y que satisface las expectativas de la población Tacneña en general.

Cuadro $\mathrm{N}^{\circ} 5$ Distribución según percepción que tiene la sociedad con respecto a la calidad de Educación que brinda la UNJBG Tacna 2008

\begin{tabular}{|c|c|c|c|c|}
\hline \multirow{2}{*}{\multicolumn{2}{|c|}{ Calidad de Educación }} & \multicolumn{2}{|c|}{ Respuestas } & \multirow{2}{*}{$\begin{array}{c}\text { Porcentaje de } \\
\text { casos } \\
\mathrm{N}^{\mathrm{j}}\end{array}$} \\
\hline & & $\mathrm{N}^{\mathrm{N}}$ & Porcentaje & \\
\hline Desacuerdo & 1 & 14 & $0,7 \%$ & $3,6 \%$ \\
\hline Parcialmente de acuerdo & 2 & 285 & $14,8 \%$ & $74,2 \%$ \\
\hline De acuerdo & 3 & 720 & $37,5 \%$ & $187,5 \%$ \\
\hline Muy de acuerdo & 4 & 598 & $31,1 \%$ & $155,7 \%$ \\
\hline Totalmente de acuerdo & 5 & 303 & $15,8 \%$ & $78,9 \%$ \\
\hline Total & & 1920 & $100,0 \%$ & $500.0 \%$ \\
\hline
\end{tabular}




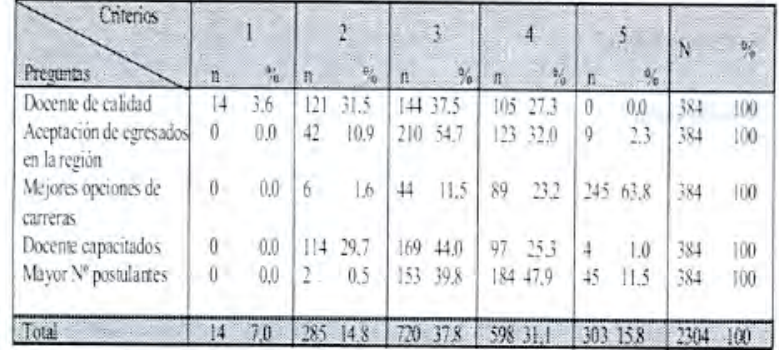

Fuente: Encuesta Dic. 2008-Febrero 2009

\section{Interpretación}

En relación a la percepción de la calidad de Educación que brinda la Universidad Nacional Jorge Basadre Grohmann, en su gran mayoría está de acuerdo que es de buena calidad representada por el $37,8 \%$, seguida del 31,1 que está muy de acuerdo, y el 15,8 está totalmente de acuerdo, el 14,8\% está parcialmente de acuerdo y el $7,0 \%$ está en desacuerdo.

Cuadro $\mathrm{N}^{\circ} 6$ Distribución según la percepción de que la UNJBG asegura la formación profesional Tacna 2008

\begin{tabular}{|c|c|c|c|c|}
\hline \multirow{2}{*}{\multicolumn{2}{|c|}{ Asegura fommación profesional }} & \multicolumn{2}{|c|}{ Respuestis } & \multirow{2}{*}{$\begin{array}{c}\begin{array}{c}\text { Porcentaje de } \\
\text { casos }\end{array} \\
\mathrm{N}^{0}\end{array}$} \\
\hline & & $\mathrm{N}^{*}$ & Porcentaje & \\
\hline Desacuerdo & 1 & 122 & $6.4 \%$ & $31,8 \%$ \\
\hline Parcialmente de acuerdo & 2 & 231 & $12,0 \%$ & $60,2 \%$ \\
\hline De acuerdo & 3 & 661 & $34,4 \%$ & $172.1 \%$ \\
\hline Muy de acuerdo & 4 & 755 & $39,3 \%$ & $196,6 \%$ \\
\hline Totalmente de acuerdo & 5 & 151 & $7,9 \%$ & $39,3 \%$ \\
\hline Total & & 1920 & $100,0 \%$ & $500,0 \%$ \\
\hline
\end{tabular}

\begin{tabular}{|c|c|c|c|c|c|c|c|c|c|c|c|c|}
\hline \multirow{2}{*}{ Progumtas } & \multicolumn{2}{|c|}{1} & \multicolumn{2}{|r|}{2} & \multicolumn{2}{|r|}{3} & \multicolumn{2}{|r|}{4} & \multicolumn{2}{|r|}{5} & \multirow{2}{*}{ N } & \multirow{2}{*}{$\%$} \\
\hline & & $\%$ & & $\begin{array}{l}\mathrm{n} \\
\% \\
\%\end{array}$ & A & $\%$ & $\pi$ & $\%$ & $n$ & $\%$ & & \\
\hline Docente de calidad & & 3,6 & & 31,5 & & 375 & 105 & 273 & 0 & 0,0 & 384 & 100 \\
\hline $\begin{array}{l}\text { Aceptación de egresados } \\
\text { en la región }\end{array}$ & 0 & 0,0 & $\begin{array}{l}42 \\
10,9\end{array}$ & & 210 & 54,7 & 123 & 32,0 & 9 & 2,3 & 384 & 100 \\
\hline $\begin{array}{l}\text { Mejores opciones de } \\
\text { carteras }\end{array}$ & 0 & 0,0 & 6 & & 44 & 11.5 & 89 & 232 & 245 & 63,8 & 384 & 100 \\
\hline Docente capacitados & 0 & 0,0 & 1,6 & & 169 & 44,0 & 97 & 253 & 4 & 1,0 & 384 & 100 \\
\hline Mayor $N^{p}$ postulartes & 0 & 0,0 & & $\begin{array}{r}29,7 \\
0,5\end{array}$ & 153 & 39.8 & 184 & 47,9 & 45 & 11,5 & 384 & 100 \\
\hline Total & 14 & 70 & 225 & 14.8 & 720 & 37,8 & 598 & 31.1 & 303 & 15.8 & 2304 & 100 \\
\hline
\end{tabular}

Fuente: Encuesta Dic. 2008-Febrero 2009

\section{Interpretación}

La percepción que tiene la sociedad en relación a que la UNJBG asegura la formación profesional, están muy de acuerdo en el 39,9\%, el 34,4\% está de acuerdo, el 12,2\% está parcialmente de acuerdo, el 7,9\% está totalmente de acuerdo y el $6,4 \%$ está en desacuerdo.
Cuadro $\mathrm{N}^{\circ} 7$ Distribución segun opciones para postulación a la UNJBG Tacna 2008

\begin{tabular}{|c|c|c|c|c|}
\hline \multirow{2}{*}{\multicolumn{2}{|c|}{$\begin{array}{l}\text { Opciones para postufacion a ta } \\
\text { UNJBG }\end{array}$}} & \multicolumn{2}{|c|}{ Resp atestas. } & \multirow{2}{*}{$\begin{array}{c}\text { Porcentajede } \\
\text { easos } \\
\mathrm{N}^{0}\end{array}$} \\
\hline & & $\mathrm{N}^{\prime \prime}$ & Porcentaje & \\
\hline Desacuerdo & 1 & 26 & $1.4 \%$ & $6.8 \%$ \\
\hline Pareialmente de acuerdo & 2 & 244 & $12.7 \%$ & $63.5 \%$ \\
\hline De acuerdo & 3 & 609 & $31,7 \%$ & $158.6 \%$ \\
\hline Muy de acuerdo & 4 & 743 & $38.7 \%$ & $193.5 \%$ \\
\hline Totalmente de acuerdo & 5 & 298 & $15,5 \%$ & $77.6 \%$ \\
\hline Total & & 1920 & $100.0 \%$ & $500.0 \%$ \\
\hline
\end{tabular}

\begin{tabular}{|c|c|c|c|c|c|c|c|c|c|c|c|c|}
\hline Chitcrios & & & & 2 & & $\overline{3}$ & & 4 & & 5 & & \\
\hline Pregratas & $\mathrm{n}$ & $\%$ & $n$ & $\%$ & $\mathrm{n}$ & $\%$ & $\mathrm{n}$ & $\%$ & $\mathrm{n}$ & $\%$ & & \\
\hline Organización/UNIBG & 18 & 4,7 & 95 & 24,7 & 185 & $4 \times 2$ & 74 & 19.3 & 12 & 3,1 & 384 & 100 \\
\hline $\begin{array}{l}\text { Carple desartilo } \\
\text { aadénion }\end{array}$ & 6 & 1.6 & 110 & 28,6 & 157 & 40.9 & 99 & 25.8 & 12 & 3.1 & 384 & 100 \\
\hline $\begin{array}{l}\text { Estabilichd atcridxds } \\
\text { Politica mass a }\end{array}$ & 1 & 03 & 8 & 21 & 46 & 120 & 173 & 45.1 & 156 & 40.6 & 384 & 100 \\
\hline esenfartes & 0 & 000 & 2 & 0.5 & 49 & 128 & 261 & 680 & 72 & 18.8 & 384 & 100 \\
\hline Tote: & 26 & 1,4 & 344 & & 609 & 31,2 & 743 & 38,7 & $x$ & 15,5 & 2304 & 100 \\
\hline
\end{tabular}

Fuente: Encuesta Dic. 2008-Febrero 2009

\section{Interpretación}

Según perciben como opción para postular a la UNJBG, consideran que sus componentes tienen un rol directo para su funcionamiento, el $38,7 \%$ está muy de acuerdo, el $31,7 \%$ está de acuerdo, el $15,5 \%$ está totalmente de acuerdo, el $12,7 \%$ parcialmente de acuerdo y el 1,4\% está en desacuerdo.

\section{DISCUSIÓN}

Las edades de ingreso a la Universidad Nacional Jorge Basadre Grohmann fluctúan entre los 17 a 19 años, haciendo un bloque porcentual de 87,5; en menor porcentaje encontramos edades por debajo o por encima de estas edades. En relación con el sexo, vemos que en un porcentaje no muy diferencial, tenemos que el $59,9 \%$ está conformado por el sexo masculino, y el $40,1 \%$ por sexo femenino.

Podemos inferir que en la UNJBG existen Facultades que predominantemente son de particular preferencia por el sexo masculino, como el caso de las Ingenierías, que a su vez podrían señalar la diferencia porcentual, sin que ello signifique que no pueda acceder un estudiante de sexo femenino.

Entre las Facultades más numerosas, tenemos FACE, FACI y FCAG, FACA y FLCJ, por lo que la muestra es mayor en relación con las otras. También el número de escuelas profesionales es 3 ó más en comparación a otras que poseen 2 ó menos escuelas.

En el Cuadro $\mathrm{N}^{\circ} 4$ observamos la percepción que tiene la sociedad con respecto a la UNJBG que está dado por la imagen, prestigio, reconocimiento y confianza, seriedad y excelencia, educación de buena calidad y en la satisfacción de expectativas que tiene de la formación profesional que brindamos. El 44,1\% está de acuerdo con los ítems solicitados, el 24,0\% está muy de acuerdo y el 6,7\% totalmente de acuerdo; en menor proporción 
están parcialmente de acuerdo $17,6 \%$, y en desacuerdo el $7,5 \%$.

Como lo señala la teoría, la percepción está determinada por varios elementos, destacando el rol que desempeña el Docente, y la imagen que proyecta como Institución de Educación Superior desde su organización y función como tal, ante la sociedad que es el referente más directo y donde se insertan los profesionales que egresan de nuestra Universidad Jorge Basadre Grohmann. Este aspecto constituye una fortaleza para tomar en cuenta al momento de planificar la mejora continua de la calidad de educación.

En el Cuadro $\mathrm{N}^{\circ} 5$ sobre percepción de la calidad de Educación que brinda la UNJBG, tenemos que el $37,8 \%$ está de acuerdo, el $31,1 \%$ está muy de acuerdo, el 15,8 totalmente de acuerdo, el $14,8 \%$ parcialmente de acuerdo y el $7,0 \%$ en desacuerdo.

Sobre los criterios a través de los cuales la sociedad percibe buena calidad de educación tenemos los siguientes: personal docente de calidad, egresados son bien aceptados en la región, la Universidad ofrece variedad de carreras profesionales, cuenta con personal docente bien capacitado y cuenta con mayor número de postulantes a las diversas Facultades y escuelas profesionales, lo que nos indicaría que los 40 años de presencia que tiene la UNJBG en la región de Tacna, ha permitido ser percibida como quien aun tiene el liderazgo. Lo que nos comprometería a quienes estamos en el desempeño de funciones a mantener y aun elevar la calidad de educación que brindamos, ya que este aspecto constituye una fortaleza institucional.

En el Cuadro $\mathrm{N}^{\circ} 6$ sobre si la UNJBG asegura la formación profesional, tenemos que el $39,3 \%$ está muy de acuerdo en afirmar que así es, el $34,4 \%$ está de acuerdo, el $12,0 \%$ está parcialmente de acuerdo y el $7,9 \%$ totalmente de acuerdo.

Los criterios utilizados fueron buena infraestructura, buen equipamiento, gratuidad de la enseñanza, tiempo de formación, y experiencia institucional en la formación profesional, ante lo cual la sociedad ha respondido que la UNJBG tiene todos los elementos para garantizar una buena formación. Los componentes internos de toda Institución también provocan un impacto social lo cual es muy útil para plantear políticas de desarrollo, planteamiento y ejecución de proyectos que fortalezcan y permitan continuar el crecimiento cuantitativo como cualitativo

En el Cuadro $\mathrm{N}^{\circ} 7$ se tratan las opciones que también considera la sociedad al postular a la UNJBG. El 38,7\% está muy de acuerdo que es un aspecto a mejorar, el $31,7 \%$ está de acuerdo, el $15,5 \%$ está totalmente de acuerdo, el $12,7 \%$ parcialmente de acuerdo y en desacuerdo el $1,4 \%$.

La sociedad no ha dejado pasar por alto que por ser una institución estatal, estos últimos años la UNJBG ha venido sufriendo interrupciones por huelgas, paros, $\mathrm{u}$ otros similares, que retrasan el desarrollo académico; sin embargo este aspecto si nos llama a considerar estrategias para revertir tal situación, que las reivindicaciones profesionales e institucionales no nos opaquen para ver en un solo sentido sino a desarrollar estrategias creativas que fortalezcan la unidad dentro de la democracia que caracteriza a la Universidad.

Para obtener un buen resultado de la labor frente a la sociedad, los docentes, debemos hacer un análisis de todos los conceptos que intervienen en el desarrollo de la labor educativa, con la finalidad de buscar la manera de conseguir mejores logros con la gestión del docente frente a los estudiantes y sociedad tacneña.

\section{CONCLUSIONES}

1. La percepción que la sociedad estudiantil tiene de la calidad educativa es de buena a muy buena, existiendo un $21,8 \%$ que opina parcialmente de acuerdo y en desacuerdo.

2. Entre los aspectos que motivan la postulación de estudiantes a la UNJBG, primordialmente consideran la trayectoria y experiencia en la formación profesional, gratuidad de la enseñanza, aceptación en la región, docentes bien capacitados y que cuentan con infraestructura y equipamiento.

3. La percepción de calidad educativa guarda una relación directa con la postulación a la UNJBG, existiendo áreas a considerar como la organización, estabilidad de autoridades, huelgas y politica por mejorar manifestado por el $70,4 \%$ que está muy de acuerdo y de acuerdo.

4. La hipótesis planteada está sustentada por la 1ra. y 2da conclusión, que dan como resultado calidad educativa buena y elegible entre las opciones de postulación.

\section{RECOMENDACIONES}

Tenemos, en opinión de la sociedad todos los elementos para constituirnos en el referente más competitivo en relación con otras universidades locales y regionales, pues contamos con una percepción que nos favorece en cuanto a calidad educativa, elementos que aseguran la formación profesional, así como docentes capacitados y con experiencia en la formación profesional, lo cual debemos aprovechar al máximo para asegurar nuestra vigencia en la sociedad.

\section{REFERENCIAS BIBLIOGRÁFICAS}

BIGGS, John. Calidad del aprendizaje universitario. Australia, 2006.

FARRO CUSTODIO, Francisco.(2005) Cómo formular un Plan Estratégico de Universidades $e$ Institutos de Educación Superior. España, p. 117-132. HERNANDEZ DÍAZ, Fabio. (2000) Metodologia del estudio universitario. Colombia, Edit. Mc Graw Hill. HUME, David. (2005) Tratado de la naturaleza humana. Madrid Edit. TECNOS.

PRIETO NAVARRO, Leonor. (2006) Auto eficacia del profesor universitario. España: Edit. Ostes. 